\title{
New-onset cardiovascular risk factors following liver transplantation: A cohort analysis in Singapore
}

\author{
Xiao Ying $\underline{\operatorname{Li}}{ }^{1}{ }^{\text {MNutrDiet }}$, Hiang Keat $\underline{\operatorname{Tan}}{ }^{2}{ }_{\text {MRCP (UK), }}$, Yet Hua Loh ${ }^{1}$ PhD
}

\begin{abstract}
Introduction: The aims of this study were to establish weight change, incidence of non-alcoholic fatty liver disease (NAFLD) and cardiovascular risk factors (CvRF) in liver transplant recipients (LTRs).

Methods: Eighty-three patients whose mean (standard deviation [SD]) age was 55.6 (8.4) years (median follow-up 73 months) and who underwent their first liver transplantation (LT) at Singapore General Hospital between February 2006 and March 2017 were included in the study. Anthropometric, clinical and demographic data were collected retrospectively from patients' medical records. Diabetes mellitus (DM), hyperlipidaemia and hypertension were regarded as CvRF.

Results: Compared to baseline, mean (SD) body weight decreased significantly at 1 month post-LT (60.8kg [11.9] versus $64.3 \mathrm{~kg}$ [13.7], $P<0.001)$. There was a gradual recovery of body weight thereafter, increasing significantly at year $2(64.3 \mathrm{~kg}$ [12.3] vs $61.5 \mathrm{~kg}$ [13.7], $P<0.001)$ until year $5(66.9 \mathrm{~kg}$ [12.4] vs $62.2 \mathrm{~kg}$ [13.9], $P<0.001)$, respectively. The prevalence of CvRF was significantly higher post-LT. NAFLD occurred in $25.3 \%$ of LTRs and it was significantly associated with post-LT DM and hyperlipidaemia.

Conclusion: CvRF increased significantly post-LT, and NAFLD occurred in $25.3 \%$ of LTRs. Body weight dropped drastically within the first month post-LT, which then returned to baseline level just before the end of first year. This novel finding suggests that nutritional intervention needs to be tailored and individualised, based on events and time from transplant. Although long-term obesity is a significant problem, aggressive oral or enteral nutritional supplements take precedence in the early and immediate post-LT period, while interventions targeted at metabolic syndrome become necessary after the first year.
\end{abstract}

Ann Acad Med Singap 2021;50:548-55

Keywords: Cardiovascular risk factors, liver transplantation, non-alcoholic fatty liver disease, obesity, weight change

\section{INTRODUCTION}

Malnutrition is well described in liver cirrhosis. The hyperdynamic circulation and altered metabolism in cirrhosis result in a hypercatabolic state which accelerates tissue breakdown. ${ }^{1}$ Ascites and gastrointestinal dysmotility in advanced cirrhosis, compounded by the need for dietary restrictions, often result in early satiety and anorexia. ${ }^{2}$ Inevitably, liver transplant wait-list patients eat poorly, leading to a diet grossly deficient in calories, protein and nutrients, culminating in muscle wasting and weight loss. ${ }^{2}$

Liver transplantation (LT) is the only curative treatment for end-stage liver failure. ${ }^{3}$ After LT, patients regain a sense of well-being and a more liberalised diet contribute to improved appetite. ${ }^{4} \mathrm{~A}$ higher caloric intake, the effect of tacrolimus associated with hypometabolism, ${ }^{4}$ increased adiposity and decreased lean muscle mass predispose liver transplant recipients (LTRs) to developing metabolic syndrome (MetS). ${ }^{5}$ More than half of long-term LT survivors will eventually develop 1 or more metabolic diseases, including obesity, impaired glucose tolerance and hypertension. $3 ., 6,7$ It is therefore not surprising that non-alcoholic fatty liver disease (NAFLD), a hepatic manifestation of MetS, is common following LT. ${ }^{8}$ A recent study by National University Hospital in Singapore showed that MetS increased significantly post-LT with a prevalence of $35.6 \% .^{9}$ However, for Singapore LTRs,

\footnotetext{
' Department of Dietetics, Singapore General Hospital, Singapore

${ }^{2}$ Department of Gastroenterology and Hepatology, Singapore General Hospital, Singapore

Correspondence: Ms Xiao Ying Li, Department of Dietetics, Singapore General Hospital, Level 10, SingHealth Tower, 10 Hospital Blvd, Singapore 168582. Email: cherrylxy2010@gmail.com
} 


\section{CLINICAL IMPACT}

\section{What is New}

- Our study reported 3-monthly body weight trends in liver transplant recipients for the first year. Weight reduced significantly by $5.5 \%$ in the first month and increased to baseline level before the end of 1 year.

- The recipients were followed up for a median period of 73 months. Metabolic complications increased significantly post-liver transplant and non-alcoholic fatty liver disease occurred in $25.3 \%$ of recipients.

\section{Clinical Implications}

- Oral or enteral nutritional supplements take precedence in the early post-transplant period, while interventions targeted at metabolic syndrome are necessary after the first year.

the evolution of body weight (BW) and the development of NAFLD post-LT remain unclear. We aimed to establish weight change, prevalence of NAFLD and cardiovascular risk factors (CvRF) in stable LTRs in Singapore.

\section{METHODS}

This was a retrospective study of adult patients who underwent LT at Singapore General Hospital between February 2006 and March 2017. The study, approved by the Institutional Review Board, included patients older than 21 years of age at the time of their transplantation, and with at least 1 year of post-LT follow-up. Exclusion criteria were multiorgan transplantation and re-transplantation. A retrospective chart review was conducted for eligible study participants. Demographic, anthropometric, clinical and biochemical data were collected. Demographic characteristics included age, sex and ethnicity. Clinical data included pre-LT Child-Pugh scores, Model for End-stage Liver Disease (MELD) scores and indications for LT, pre-LT and post-LT comorbidities. Weight, height and body mass index (BMI) were recorded at the time of transplant, 1 month post-LT, and at 3-monthly intervals, until 1 year post-LT and then annually thereafter. For patients with fluid overload at LT, dry weight was estimated according to Mendenhall et al. ${ }^{10}$ For those with mild, moderate and severe ascites, BW was subtracted by $2.2 \mathrm{~kg}, 6.0 \mathrm{~kg}$ and $14.0 \mathrm{~kg}$, respectively. In the case of mild, moderate and severe oedema, BW was adjusted by subtracting $1.0 \mathrm{~kg}, 5.0 \mathrm{~kg}$ and $10.0 \mathrm{~kg}$, respectively. The primary outcomes of the study were post-LT weight trends and obesity prevalence. Secondary outcomes were the incidence of NAFLD and CVRF, defined by hypertension, hyperlipidaemia and diabetes mellitus (DM).

The following definitions were used in the study:

(1) Hypertension: systolic or diastolic blood pressure $>140$ or $90 \mathrm{mmHg}$; or patient on at least 1 antihypertensive drug for more than 3 months post-LT; 11

(2) Hyperlipidaemia: low-density lipoprotein (LDL) $>2.6 \mathrm{mmol} / \mathrm{L}$ or triglyceride $(\mathrm{TG})>1.7 \mathrm{mmol} / \mathrm{L}$; or patient on statin or fibrate for more than 3 months post-LT; ${ }^{11}$

(3) DM: persistent fasting or random blood glucose level $\geq 7.0 \mathrm{mmol} / \mathrm{L}$ or $\geq 11.1 \mathrm{mmol} / \mathrm{L}$, respectively; or patient on antidiabetic drug after the first 3 months post-LT; ${ }^{11}$

(4) Post-LT NAFLD diagnosis was based on ultrasound or computerised tomography (CT) scan of the liver. ${ }^{12}$ In our centre, ultrasound surveillance on the liver graft is routinely performed 3-monthly for the first 12 months post-LT, then annually for patients without pre-LT hepatocellular carcinoma; whereas patients with hepatocellular carcinoma prior to LT are monitored with CT scan 3-monthly for the first 24 months, followed by alternating $\mathrm{CT}$ and ultrasound thereafter;

(5) Obesity: BMI $>27.5 \mathrm{~kg} / \mathrm{m}^{2}$, based on World Health Organization (WHO) recommendations for Asians. ${ }^{13}$

\section{Post-transplant care}

Our multidisciplinary LTR care involves transplant surgeons, hepatologists, endocrinologists, pharmacists, specialty nurses, dietitians and physiotherapists. We practise triple immunosuppression with corticosteroid, a calcineurin inhibitor (CNI) - preferably tacrolimusand mycophenolate mofetil. Tacrolimus dose is titrated to a trough of $7-10 \mathrm{ng} / \mathrm{mL}$ in the first 3 months, $5-7 \mathrm{ng} / \mathrm{mL}$ in months $4-6$, and thereafter $4-6 \mathrm{ng} / \mathrm{mL}$. Corticosteroid dose is gradually tapered and stopped after 3 months. In patients with pre-existing renal impairment, additional induction with basiliximab is considered to delay exposure to CNI at reduced dose. Lipid profile and glycated haemoglobin (HbAlc) are monitored every 3-6 months. Pharmacological therapies are initiated for suboptimal levels by endocrinologists. 


\section{Statistical analysis}

Statistical analysis was performed using SPSS Statistics software version 25 (IBM Corp, Armonk, US). Continuous variables were expressed as mean and standard deviation (SD) and categorical variables as frequencies and percentages (\%). Statistical significance of mean difference between continuous variables was determined by paired-samples $t$ test. Chi-square test was performed for categorical variables. Prevalence of CvRF preand post-LT were compared by McNemar's test. The statistical significance level was set at $P<0.05$.

\section{RESULTS}

From February 2006 to March 2017, 99 patients underwent LT. Included in our study were 83 LTRs, as 16 LTRs were excluded due to early death $(\mathrm{n}=15)$ and re-transplantation $(\mathrm{n}=1)$. Table 1 shows the baseline characteristics of the study population. The mean (SD) age was $55.6(8.4)$ years and the majority were Chinese (83.1\%). The median duration of post-LT follow-up was 73 (interquartile range [IQR] 34-109) months. The most common indication for LT was hepatitis B (44.6\%). The mean (SD) MELD score at LT was 18 (8) and a majority were of Child-Pugh class C (43.4\%). Most of the LTRs (90.4\%) received tacrolimus for immunosuppression.

\section{Obesity and body weight change}

Post-LT obesity prevalence was $24.1 \%$, which was higher than pre-LT $(18.1 \%, P<0.05)$. Post-LT obesity was associated with pre-LT obesity $(P<0.0001)$, with $66.7 \%$ of patients with pre-existing obesity remaining obese post-LT. There were 10 LTRs (14.7\%) who developed de novo obesity post-LT, at a median (IQR) of 17 (9-45) months. Fig. 1 shows post-LT BW trend. Compared to baseline, mean (SD) BW decreased significantly at 1 month post-LT (60.8kg [11.9] versus $64.3 \mathrm{~kg}$ [13.7], $P<0.001)$. This was followed by a gradual recovery of $\mathrm{BW}$ which eventually reached pre-LT level at 9 months post-LT. Mean (SD) weight gain continued until the fifth year $(66.9 \mathrm{~kg}[12.4] \mathrm{vs}$ $62.2 \mathrm{~kg}[13.9], P<0.001)$.

\section{Cardiovascular risk factors}

At last follow-up in March 2018, the rate of newonset DM, hypertension and hyperlipidaemia was $29.3 \%, 44.5 \%$ and $46.4 \%$, respectively (Fig. 2). A higher prevalence for DM $(68.9 \%$ vs $57.8 \%$, $P<0.005)$, hypertension $(62.7 \%$ vs $33.7 \%, P<0.0001)$, and hyperlipidaemia $(57.8 \%$ vs $28.9 \%, P<0.0001)$ was observed post-LT. There was no significant
Table 1. Baseline characteristics of liver transplant recipients

\begin{tabular}{|c|c|}
\hline Characteristics & $\mathrm{n}=\mathbf{8 3}$ \\
\hline Age, mean (SD), years & $55.6(8.4)$ \\
\hline Male, no. (\%) & $57(68.7)$ \\
\hline Female, no. (\%) & $26(31.3)$ \\
\hline \multicolumn{2}{|l|}{ Ethnicity, no. (\%) } \\
\hline Chinese & $69(83.1)$ \\
\hline Malay & $5(6.0)$ \\
\hline Indian & $4(4.8)$ \\
\hline Others $^{\mathrm{a}}$ & $5(6.0)$ \\
\hline \multicolumn{2}{|l|}{ Time from transplant } \\
\hline Mean (SD), months & $74.5(38.9)$ \\
\hline Median (IQR), months & $73.0(34.0-109.0)$ \\
\hline \multicolumn{2}{|c|}{ Aetiologies of liver diseases, no. $(\%)^{b}$} \\
\hline $\mathrm{HBV}$ & $37(44.6)$ \\
\hline NASH/cryptogenic cirrhosis & $15(18.1)$ \\
\hline PBC, PSC, AIH & $14(16.9)$ \\
\hline ALD & $8(9.6)$ \\
\hline $\mathrm{HCV}$ & $5(6.0)$ \\
\hline Others & $11(13.3)$ \\
\hline MELD score, mean (SD) & $18(8)$ \\
\hline \multicolumn{2}{|l|}{ Child-Pugh score, no. (\%) } \\
\hline A & $15(18.0)$ \\
\hline B & $32(38.6)$ \\
\hline $\mathrm{C}$ & $36(43.4)$ \\
\hline
\end{tabular}

AIH: autoimmune hepatitis; ALD: alcoholic liver disease; HBV: hepatitis B virus; HCV: hepatitis C virus; MELD: Model for End-stage Liver Disease; NASH: non-alcoholic steatohepatitis; PBC: primary biliary cholangitis; PSC: primary sclerosing cholangitis; SD: standard deviation

a Other aetiologies included acute liver failure (6 cases), familial amyloid polyneuropathy ( 2 cases), type 2 citrullinemia ( 2 cases) and Caroli disease (1 case)

b The total adds up to more than $100 \%$ as some patients had more than 1 aetiologies for chronic liver disease or indications for liver transplantation

difference for $\mathrm{HbAlc}$ between baseline and any time point post-LT, while total cholesterol (T-CHOL), highdensity lipoprotein (HDL), TG and LDL increased significantly 3 months post-LT (Fig. 3). However, the mean values of T-CHOL, HDL and TG were all within healthy range at all time points. LDL levels were slightly above the desirable level of $2.6 \mathrm{mmol} / \mathrm{L}$ at 3 months, 6 months and 3 years. 


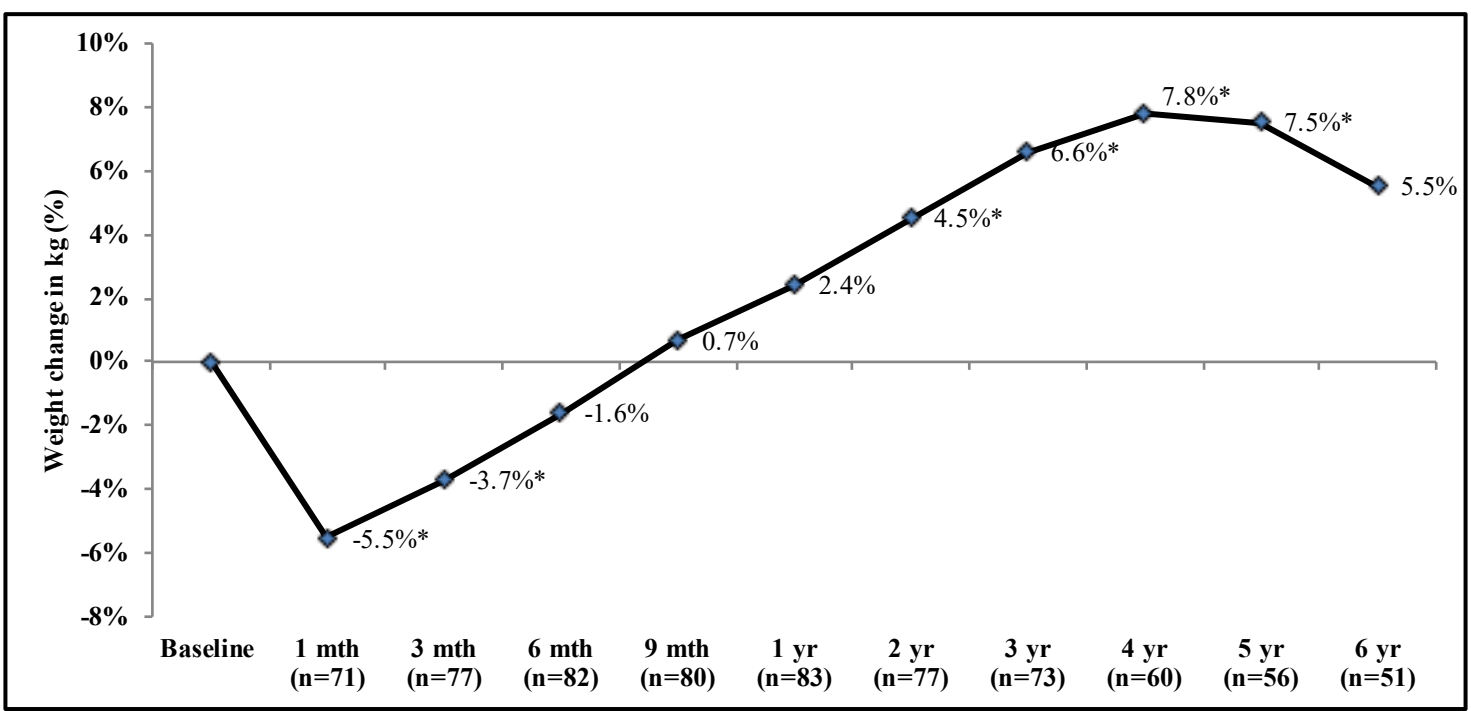

Fig. 1. Body weight change in liver transplant recipients post-liver transplantation. $* P<0.01$

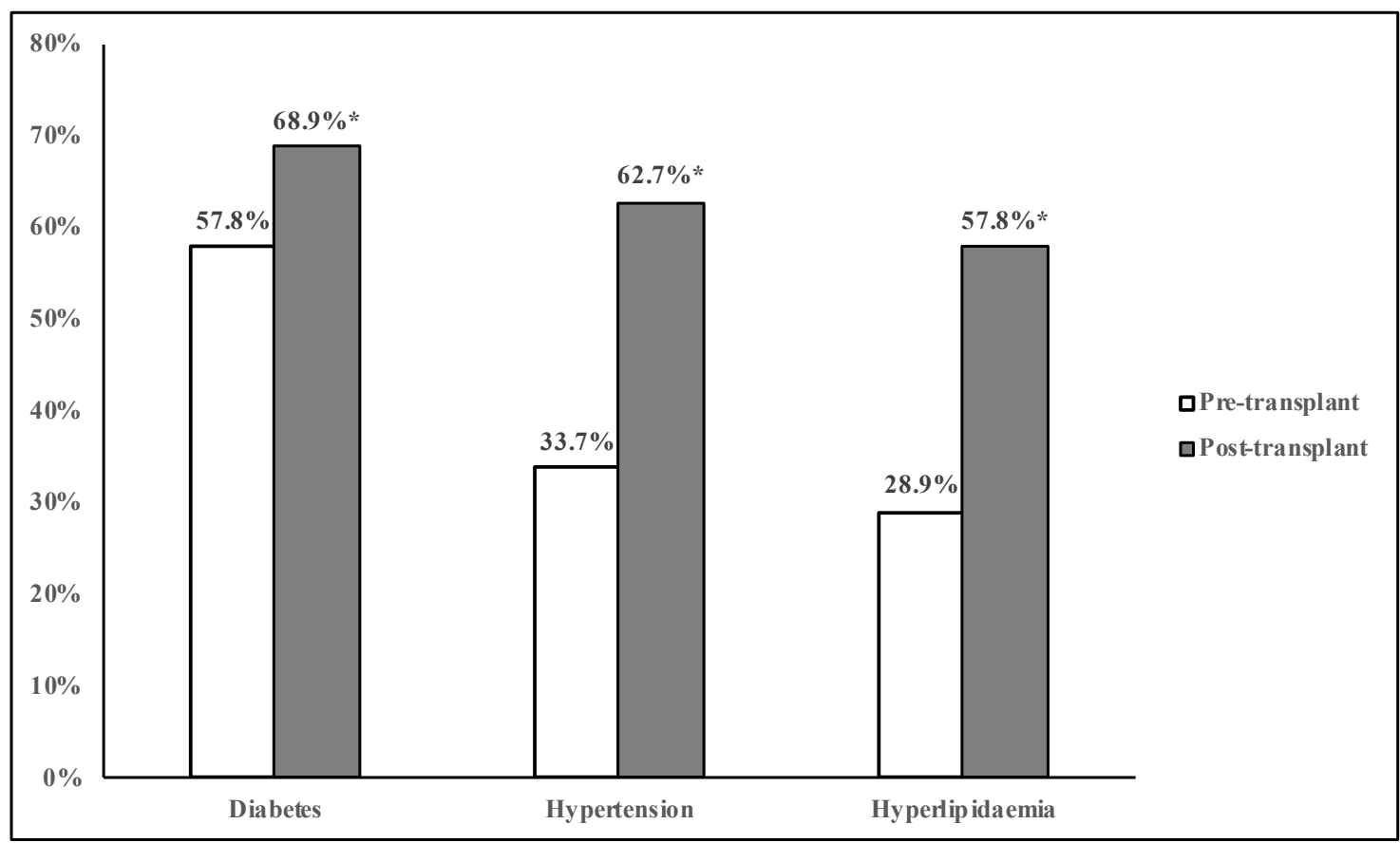

Fig. 2. Prevalence of cardiovascular risk factors pre- and post-transplantation. $* P<0.001$

NAFLD occurred in 21 patients $(25.3 \%)$, at a median (IQR) of 16 (10-37) months post-LT. Approximately $21 \%$ developed de novo NAFLD post-LT. Interestingly, almost half $(46.7 \%)$ of the patients with a history of cryptogenic or non-alcoholic steatohepatitis (NASH) cirrhosis developed post-LT NAFLD. Overall, postLT NAFLD was associated with DM $(91.4 \%$ patients, $P<0.01)$ and hyperlipidaemia $(85.3 \%$ patients, $P<0.005$ ). Although $33.2 \%$ post-LT NAFLD patients were obese, we did not find any statistically significant association between post-LT NAFLD and obesity.

\section{DISCUSSION}

Our study showed that BW and the prevalence of CvRF increased significantly in LTRs, including the occurrence of NAFLD. This suggests that the burden of obesity and MetS is high among LTRs in Asia. The consequence of MetS is significant and our study 


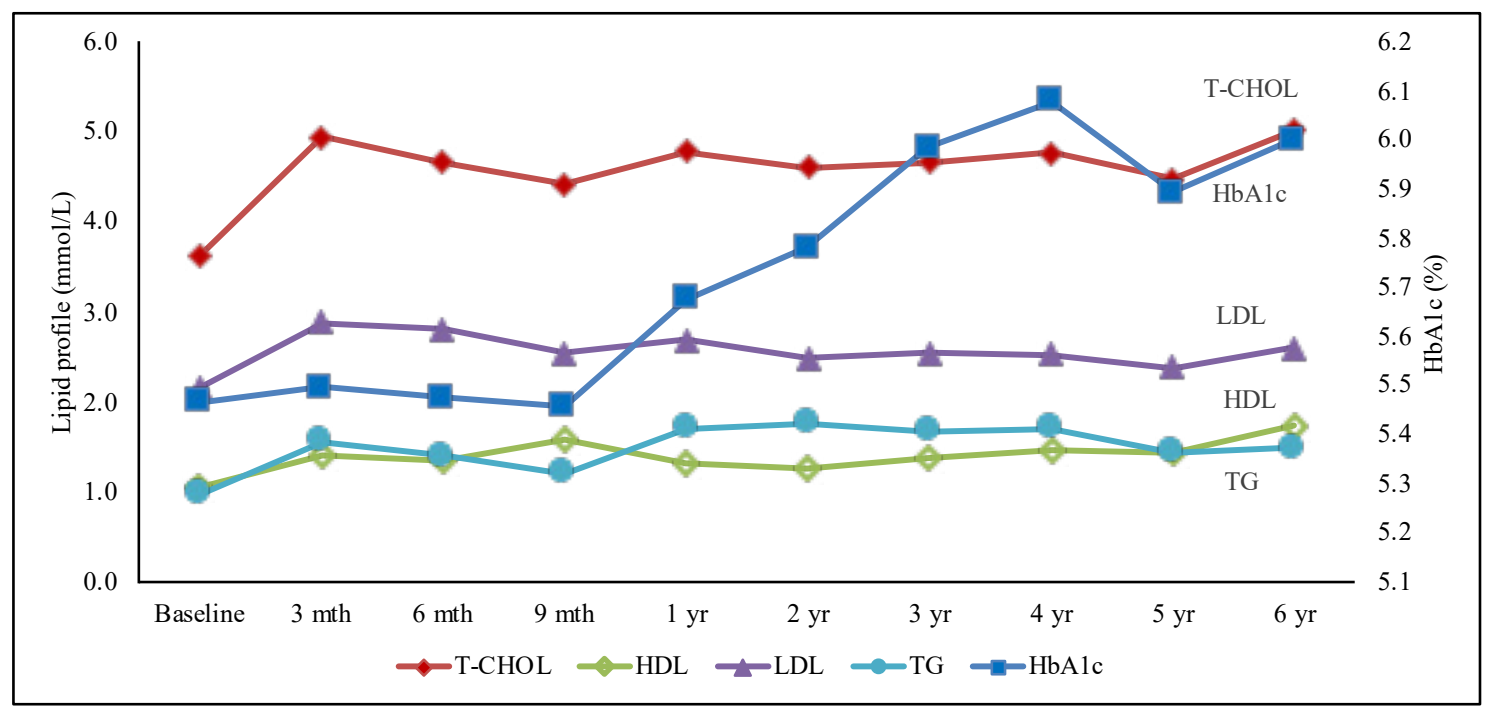

Fig. 3. Trends of HbA1c and lipid profile post-liver transplantation.

HDL: high-density lipoprotein; LDL: low-density lipoprotein; TG: triglyceride; T-CHOL: total cholesterol

highlights the multiple cardiovascular risks in this population.

We collected BW data at multiple time points post-LT and reviewed patients for more than 6 years. To the best of our knowledge, this is the first study reporting detailed BW change in the early post-LT period at close intervals, with the longest follow-up duration. Other studies reported weight trends with either less frequent intervals or shorter follow-up duration. ${ }^{6,14}$ The weight trend in our study offers fresh insights into the dynamic weight change of LTRs, especially during the early and intermediate post-LT period where early post-surgery catabolism frequently necessitates oral nutritional supplements or tube feeding to prevent weight loss. Later excessive weight gain from effects of immunosuppression and improved appetite requires timely nutritional intervention to curb obesity and prevent metabolic complications in the longer term.

A majority of studies in the literature did not report weight change at 1 month post-LT. ${ }^{6,14,15}$ A significant weight loss at 1 month after transplantation has been reported, due in part to a reduced fluid overload. ${ }^{16}$ Another major factor contributing to the weight loss is the hypercatabolic state in the immediate post-LT period, resulting in increased muscle breakdown and loss. A longitudinal study showed that resting energy expenditure increased significantly post-surgery to as high as $42 \%$ above predicted value, peaking at day 10 , and that hypermetabolism persisted for 6 months only to resolve at 1 year post-LT. ${ }^{17}$ Furthermore, patients were typically fasted in the immediate postoperative period until they were able to pass flatus. By the time a full diet was allowed, a few days would have typically passed. This would represent missed opportunities for early postoperative tube feeding within 12 hours, which has been shown to be beneficial in reducing length of stay in intensive care and in lowering infection rates. ${ }^{18}$ Additionally, intermittent fasting is often necessary for post-surgery protocol ultrasound scans (for early detection of hepatic artery thrombosis) and other advanced imaging procedures (in patients suspected of having biliary leaks or strictures). ${ }^{19,20}$ These frequent interruptions in the delivery of nutrition to LTRs in the early postoperative period pose a major challenge to maintaining a delicate balance between fasting for investigations and provision of adequate calories and protein to an already malnourished population.

After the initial decrease in the first month, the weight of our LTRs gradually increased. This was partially due to the hypermetabolic state slowly regressing to normal, resulting in a lower energy expenditure compared to the period immediately post-LT. ${ }^{17}$ The disappearance of ascites after LT leads to a marked improvement in patients' appetite and food intake. ${ }^{21}$ Richardson et al. reported that mean (SD) calorie intake in post-LT patients increased significantly at $2,227 \mathrm{kcal} / \mathrm{d}$ (41) compared with consumption at $1,542 \mathrm{kcal} / \mathrm{d}$ (124) during the pre-transplant period. ${ }^{21}$ Our study showed that BW eventually returned to pre-LT level around 9 months post-LT. Weight gain continued to progress, reaching a peak in the fourth year. Two large-scale studies by Everharts et al. and 
Richards et al. showed that the most rapid weight gain occurred within the first year after LT, but the initial drastic weight loss seen in our study at 1 month was not reported in the studies due to a lack of data in the early postoperative period. . $^{6,14}$

Similar to the study by Tan et al., ${ }^{9}$ nearly a quarter (24.1\%) of our LTRs was found to have obesity after a median follow-up of 73 months. This was comparatively lower than the prevalence of $30 \%$ reported by the aforementioned 2 studies. ${ }^{6,14}$ This discrepancy could possibly be due to the difference in the trajectory of BW change during the first year post-LT. While our patients suffered from significant weight loss in the first month before a gradual recovery in BW to baseline level, the other 2 studies reported rapid weight gain of $5-6 \mathrm{~kg}$ at 1 year postLT. Of note, we defined obesity at BMI cut-off of $27.5 \mathrm{~kg} / \mathrm{m}^{2}$ based on WHO recommendations, ${ }^{13}$ as opposed to BMI of $30.0 \mathrm{~kg} / \mathrm{m}^{2}$ in many Western studies. ${ }^{6,14}$ In spite of differences in definition, we believe that our study reflects the true health risks of our LTRs as it is well established that Asians have significantly higher risks for type $2 \mathrm{DM}$ and cardiovascular diseases at a lower BMI compared to their Caucasian counterparts. ${ }^{13}$ Similar to most Western studies, ${ }^{6,14,22}$ our centre's post-LT obesity prevalence is significantly higher than the general local population. Based on recent data from the Ministry of Health, Singapore, obesity prevalence was $8.7 \%$ in local adult residents. ${ }^{23}$ Newly developed obesity was $14.7 \%$ in our LTRs, compared with $15.5-40.7 \%$ reported in other studies. ${ }^{6,24}$

In our study, the prevalence of DM, hypertension and hyperlipidaemia was $68.9 \%, 62.7 \%$ and $57.8 \%$, respectively. Other centres reported $61 \%,{ }^{22} 62 \%{ }^{22}$ and $27-71 \%,{ }^{25}$ respectively, in comparison with Tan et al. ${ }^{9}$ who reported $51.1 \%, 60.0 \%$ and $46.7 \%$, respectively. The incidence of new-onset DM, hypertension and hyperlipidaemia in our study was $29.3 \%, 44.5 \%$ and $46.4 \%$, respectively and this was comparable with other studies, ${ }^{26-28}$ where post-LT prevalence was much higher than pre-LT figures. It is widely believed that immunosuppressive drugs play a crucial role in the onset of metabolic complications post-LT. Steroid increases insulin resistance and reduces beta cell function; influencing glucose homeostasis and contributing to the development of DM. ${ }^{27}$ It also increases vascular resistance and cardiac contractility, leading to hypertension. ${ }^{27}$ Steroids induce hyperlipidaemia via the stimulation of enzyme activity involved in cholesterol metabolism and fatty acid synthesis. ${ }^{29}$ New onset diabetes may also be induced by CNI reducing insulin secretion. ${ }^{30}$ Furthermore, the vasoconstrictive effect of CNI on renal arteriole induces sodium and water reabsorption with a consequence of volume expansion, contributing to blood pressure increase..$^{31}$ Lastly, CNI reduces cholesterol excretion through the biliary system and blocks LDL-cholesterol receptors, resulting in elevated blood cholesterol levels. ${ }^{32}$

Although the prevalence of CvRF was high in our cohort, control of hyperlipidaemia and DM was excellent. One of the current leading causes of mortality among long-term transplant survivors is cardiovascular diseases. ${ }^{33}$ Mean values of T-CHOL, HDL and TG were all well within healthy ranges at all time points. The highest mean $\mathrm{HbAlc}(\mathrm{SD})$ recorded was $6.1 \%(1.3)$ at 4 years post-LT, while mean (SD) LDL was $2.7 \mathrm{~mol} / \mathrm{L}(0.9)$ at 1 year post-LT. This was likely due to the intensive surveillance and management of metabolic diseases in our centre. Due to the alarmingly high prevalence of post-LT obesity, it is of paramount importance to prevent and manage obesity to improve long-term survival. As such, long-term care of our LTRs is provided for by a dedicated transplant endocrinologist and dietitian.

NAFLD, a hepatic manifestation of MetS, occurred in $25.3 \%$ of our patients. Post-transplant NAFLD recurred in almost half of patients whose indication for transplant was cryptogenic or NASH cirrhosis. In the literature, the reported recurrence ranged from $25-100 \%$, up to 5 years post-LT. ${ }^{34-37}$ De novo post-LT NAFLD was found in $20.6 \%$ of our patients. This is comparable to the incidence rate of $18 \%$ at 28 months post-LT that was reported by Seo et al. ${ }^{38}$ for 68 patients with liver biopsy. However, 2 other larger studies by Dumortier et al. and Galvin et al. reported more than $30 \%$ de novo biopsy-proven NAFLD at 3 years postLT. ${ }^{39,40}$ The varying rates are likely due to the different diagnostic criteria among the studies. Seo et al. defined de novo NAFLD as $>33 \%$ increase in hepatic steatosis compared to their respective donor biopsies, ${ }^{38}$ while Dumortier et al. and Galvin et al. adopted the more conventional diagnostic criteria of steatosis $>5 \%$ on liver biopsy. ${ }^{39,40}$ In our centre, routine protocol biopsy is not carried out except when there is a clinical suspicion of graft rejection. Although ultrasound is not sensitive enough for the detection of mild steatosis, ${ }^{12}$ it is the only practical means for the diagnosis of NAFLD. It is not surprising then that our study detected a much lower rate of de novo NAFLD compared to the literature. Reported NAFLD risk factors included increased BMI, metabolic complications, weight gain post-LT, immunosuppressant and donor liver steatosis, and use of angiotensin-converting enzyme inhibitors. ${ }^{38-40}$ 
In our study, de novo NAFLD was significantly a ssociated with post-LT DM and HLD. Data on longterm prognosis of de novo NAFLD are scarce. Galvin et al. reported that almost $40 \%$ of LTRs with de novo NAFLD had biopsies indicating significant fibrosis and $5 \%$ cirrhosis. ${ }^{40}$ Dureja et al. showed that patients with recurrent NAFLD post-LT were at a higher risk of cardiovascular diseases and death in the long term. ${ }^{36} \mathrm{We}$ were unable to examine cardiovascular mortality rate due to our small sample size and short follow-up duration.

The current study has limitations. The retrospective nature of the study would invariably introduce biases. Firstly, the actual BW in some of our pre-LT patients would have been masked by the presence of oedema/ ascites. Although we made the best effort to adjust weight based on the method described by Mendenhall et al., ${ }^{10}$ discrepancies between the estimated and actual BW may still exist. Secondly, we lacked complete data on all patients at all follow-up time points, except at 1 year post-LT. Thirdly, the diagnosis of NAFLD in our LTR cohort based on ultrasound was likely an underestimate, as ultrasound is only reliable when there is at least moderate hepatic steatosis. ${ }^{12}$ Last but not least, dietary and lifestyle interventions may have impacted the weight of our patients. Post-LT routine care included diet education and counselling from dietitians but may not routinely involve a physiotherapist, especially after hospital discharge. Physical activity data were unavailable to us to analyse the impact of an exercise regime in the evolution of BW post-LT. Based on the limited data, we cannot comment on the impact of our nutritional intervention on the lesser degree of weight gain in our LTRs compared to other centres. Although we reported similarly high incidences of DM, hypertension and hyperlipidaemia, we were unable to further examine the risk factors for the development of metabolic complications post-LT due to our limited study sample size.

\section{CONCLUSION}

CvRF increased significantly post-LT and NAFLD occurred in $25.3 \%$ of our LTRs. We observed a drastic decrease in weight within the first month post-LT, which then returned to baseline before the end of 1 year. This novel finding suggests that nutritional intervention needs to be tailored and individualised, based on events and time from LT. Although long-term obesity is a significant problem, aggressive oral or enteral nutritional supplements take precedence in the early and immediate post-LT period, while interventions targeted at MetS are necessary after the first year.

\section{REFERENCES}

1. Peng S, Plank LD, McCall JL, et al. Body composition, muscle function, and energy expenditure in patients with liver cirrhosis: a comprehensive study. Am J Clin Nutr 2007;85:1257-66.

2. Morando F, Rosi S, Gola E, et al. Adherence to a moderate sodium restriction diet in outpatients with cirrhosis and ascites: a real-life cross-sectional study. Liver Int 2015;35:1508-15.

3. Roberts MS, Angus DC, Bryce CL, et al. Survival after liver transplantation in the United States: a disease-specific analysis of the UNOS database. Liver Transpl 2004;10:886-97.

4. Ferreira LG, Santos LF, Anastacio LR, et al. Resting energy expenditure, body composition, and dietary intake: a longitudinal study before and after liver transplantation. Transplantation 2013;96:579-85

5. Brito-Costa A, Pereira-da-Silva L, Papoila AL, et al. Factors associated with changes in body composition shortly after orthotopic liver transplantation: the potential influence of immunosuppressive agents. Transplantation 2016;100:1714-22.

6. Richards J, Gunson B, Johnson J, et al. Weight gain and obesity after liver transplantation. Transpl Int 2005;18:461-6.

7. Anastacio LR, Lima AS, Toulson Davisson Correia MI. Metabolic syndrome and its components after liver transplantation: incidence, prevalence, risk factors, and implications. Clin Nutr 2010;29:175-9.

8. Khan RS, Newsome PN. Non-alcoholic fatty liver disease and liver transplantation. Metabolism 2016;65:1208-23.

9. Tan HL, Lim KB, Iyer SG, et al. Metabolic syndrome after a liver transplantation in an Asian population. HPB (Oxford) 2015; 17:713-22.

10. Mendenhall C. Protein-calorie malnutrition in alcoholic liver disease. In: Watson RR, Watzl B, eds. Nutrition and alcohol. Boca Raton: CRC Press; 1992:363-84.

11. American Diabetes Association. Standards of medical care in diabetes. Diabetes Care 2015;38:S8-16.

12. Hernaez R, Lazo M, Bonekamp S, et al. Diagnostic accuracy and reliability of ultrasonography for the detection of fatty liver: A meta-analysis. Hepatology 2011;54:1082-90.

13. World Health Organization. Appropriate body-mass index for Asian populations and its implications for policy and intervention strategies [published correction appears in Lancet 2004;363:902]. Lancet 2004;363:157-63.

14. Everhart JE, Lombardero M, Lake JR, et al. Weight change and obesity after liver transplantation: incidence and risk factors. Liver Transpl Surg 1998;4:285-96.

15. Anastacio LR, Ferreira LG, de Sena Ribeiro H, et al. Body composition and overweight of liver transplant recipients. Transplantation 2011;92:947-51.

16. Dashti SH, Kasraianfard A, Ebrahimi A, et al. Hemodynamic changes and early recovery of liver graft function after liver transplantation. Int J Organ Transplant Med 2020;11:1-7.

17. Plank LD, Metzger DJ, McCall JL, et al. Sequential changes in the metabolic response to orthotopic liver transplantation during the first year after surgery. Ann Surg 2001;234:245-55.

18. Hasse JM, Blue LS, Liepa GU, et al. Early enteral nutrition support in patients undergoing liver transplantation. JPEN J Parenter Enteral Nutr 1995;19:437-43.

19. Bekker J, Ploem S, de Jong KP. Early hepatic artery thrombosis after liver transplantation: A systematic review of the incidence, outcome and risk factors. Am J Transplant 2009;9:746-57. 
20. Arain MA, Attam R, Freeman ML. Advances in endoscopic management of biliary tract complications after liver transplantation. Liver Transpl 2013;19:482-98.

21. Richardson RA, Garden OJ, Davidson HI. Reduction in energy expenditure after liver transplantation. Nutrition 2001;17:585-9.

22. Laryea M, Watt KD, Molinari M, et al. Metabolic syndrome in liver transplant recipients: prevalence and association with major vascular events. Liver Transpl 2007;13:1109-14.

23. Ministry of Health. Disease burden. Available at: https://www.moh. gov.sg/resources-statistics/singapore-health-facts/disease-burden. Accessed on 26 Feburary 2020.

24. Stegall MD, Everson G, Schroter G, et al. Metabolic complications after liver transplantation. Diabetes, hypercholesterolemia, hypertension, and obesity. Transplantation 1995;60:1057-60.

25. Mells G, Neuberger J. Reducing the risks of cardiovascular disease in liver allograft recipients. Transplantation 2007;83:1141-50.

26. Honda M, Asonuma $\mathrm{K}$, Hayashida $\mathrm{S}$, et al. Incidence and risk factors for new-onset diabetes in living-donor liver transplant recipients. Clin Transplant 2013;27:426-35.

27. Jimenez-Perez M, Gonzalez-Grande R, Omonte Guzman E, et al. Metabolic complications in liver transplant recipients. World $\mathrm{J}$ Gastroenterol 2016;22:6416-23.

28. Martinez-Saldivar B, Prieto J, Berenguer M, et al. Control of blood pressure in liver transplant recipients. Transplantation 2012;93:1031-7.

29. Stegall MD, Everson GT, Schroter G, et al. Prednisone withdrawal late after adult liver transplantation reduces diabetes, hypertension, and hypercholesterolemia without causing graft loss. Hepatology 1997;25:173-7.

30. Chakkera HA, Mandarino LJ. Calcineurin inhibition and new-onset diabetes mellitus after transplantation. Transplantation 2013;95:647-52
31. Textor SC, Schwartz L, Wilson DJ, et al. Systemic and renal effects of nifedipine in cyclosporine-associated hypertension. Hypertension 1994;23:I220-4.

32. Watt KD, Charlton MR. Metabolic syndrome and liver transplantation: A review and guide to management. J Hepatol 2010;53:199-206.

33. Watt KD, Pedersen RA, Kremers WK, et al. Evolution of causes and risk factors for mortality post-liver transplant: Results of the NIDDK long-term follow-up study. Am J Transplant 2010; 10:1420-7.

34. Contos MJ, Cales W, Sterling RK, et al. Development of nonalcoholic fatty liver disease after orthotopic liver transplantation for cryptogenic cirrhosis. Liver Transpl 2001;7:363-73.

35. Yalamanchili K, Saadeh S, Klintmalm GB, et al. Nonalcoholic fatty liver disease after liver transplantation for cryptogenic cirrhosis or nonalcoholic fatty liver disease. Liver Transpl 2010;16:431-9.

36. Dureja P, Mellinger J, Agni R, et al. Nafld recurrence in liver transplant recipients. Transplantation 2011;91:684-9.

37. El Atrache MM, Abouljoud MS, Divine G, et al. Recurrence of non-alcoholic steatohepatitis and cryptogenic cirrhosis following orthotopic liver transplantation in the context of the metabolic syndrome. Clin Transplant 2012;26:E505-12.

38. Seo S, Maganti K, Khehra M, et al. De novo nonalcoholic fatty liver disease after liver transplantation. Liver Transpl 2007;13:844-7.

39. Dumortier J, Giostra E, Belbouab S, et al. Non-alcoholic fatty liver disease in liver transplant recipients: another story of "seed and soil". Am J Gastroenterol 2010;105:613-20.

40. Galvin Z, Rajakumar R, Chen E, et al. Predictors of de novo nonalcoholic fatty liver disease after liver transplantation and associated fibrosis. Liver Transpl 2019;25:56-67. 